Research Paper

\title{
Vascular Invasion and Stromal S100A4 Expression at the Invasive Front of Colorectal Cancer are Novel Determinants and Tumor Prognostic Markers
}

\author{
Tamotsu Sugai ${ }^{1}$, Noriyuki Yamada ${ }^{1}$, Makoto Eizuka ${ }^{1}$, Ryo Sugimoto ${ }^{1}$, Noriyuki Uesugi ${ }^{1}$, Mitsumasa \\ Osakabe1, Kazuyuki Ishida1 ${ }^{1}$ Kouki Otsuka², Akira Sasaki², Takayuki Matsumoto ${ }^{3 凶}$ \\ 1. Department of Molecular Diagnostic Pathology, School of Medicine, Iwate Medical University, 19-1, Morioka 020-8505, Japan; \\ 2. Department of Surgery, School of Medicine, Iwate Medical University, 19-1, Morioka 020-8505, Japan; \\ 3. Division of Gastroenterology, Department of Internal Medicine, School of Medicine, Iwate Medical University, 19-1, Morioka 020-8505, Japan. \\ $\square$ Corresponding author: Tamotsu Sugai, MD, Department of Molecular Diagnostic Pathology, Iwate Medical University, 19-1 Morioka 020-8505, Japan TEL: \\ +81-19-651-5111; FAX: +81-19-629-1437 E-mail: tsugai@iwate-med.ac.jp \\ (c) Ivyspring International Publisher. This is an open access article distributed under the terms of the Creative Commons Attribution (CC BY-NC) license \\ (https://creativecommons.org/licenses/by-nc/4.0/). See http://ivyspring.com/terms for full terms and conditions.
}

Received: 2016.12.09; Accepted: 2017.03.25; Published: 2017.06.02

\begin{abstract}
Object: The aim of the present study was to investigate the clinicopathological characteristics and prognostic factors associated with sporadic colorectal cancer (CRC). We examined the clinicopathological findings and immunohistochemical expression of tumor prognostic markers at tumor budding sites to determine their predictive value for patient prognosis.

Materials and Methods: Immunohistochemical examination was performed by tissue microarray (TMA) of specimens from 106 patients with CRC. On hematoxylin and eosin (H\&E)-stained tumor tissue slides, a representative area of tumor budding at the invasive front was selected for the construction of a TMA. Immunostaining for matrix metalloproteinase-7 (MMP7), the laminin-5 (In-5) Y2 chain and S100A4 was performed to determine the association between patient survival and these markers.

Results: Clinicopathological variables were also assessed. Tumor location, histological type, degree of lymphatic invasion and vascular invasion, tumor stage, epithelial expression of S100A4, stromal cell expression of S100A4 and expression of the In-5y2 chain were associated with an increased risk of mortality. Five factors were retained in the multivariate logistic regression analysis. Specifically, the tumor location, degree of lymphatic invasion and vascular invasion, tumor stage and stromal cell expression of S100A4 remained significant predictors of patient survival after controlling for the other variables.
\end{abstract}

Conclusion: Vascular invasion and stromal expression of S100A4 in the tumor budding areas correlated with patient survival. Stromal immunostaining of S100A4 may be useful for identifying high-risk patients with advanced CRC.

Key words: colorectal cancer, prognostic marker, S100A4, tumor budding, vascular invasion.

\section{Introduction}

The tumor node metastasis (TNM) staging system proposed by the International Union Against Cancer (UICC) is by far the most widely recommended and most reliable prognostic indicator for patients with colorectal cancer (CRC) [1, 2]. According to this system, patients with stage I disease have relatively favorable prognoses and 5-year survival rates, whereas patients with stages II, III and IV disease show progressively worse outcomes, with varying 5-year survival rates [1, 2]. However, it is well known that patients with tumors of the same TNM stage may exhibit variations in both their prognosis 
and response to therapy [2].

Pathological and molecular biomarkers, which are independent of TNM stage, have been examined for their prognostic characteristics [3, 4], and studies have shown that these parameters have promising predictive values. These prognostic factors include venous and lymphatic invasion, tumor location, tumor grade, perineural invasion, histological type and the expression of molecular markers [3-13]. In addition, recent studies have revealed that the status of the tumor invasive front plays an important role in colorectal carcinogenesis. The invasive front of CRC represents a critical interface at which tumor progression and tumor cell dissemination occur [14, 15]. Extensive investigation into the invasive front has provided a better understanding of the genetic basis of colorectal carcinogenesis and, more importantly, has identified the significance of tumor budding and the surrounding area, including cancer-associated fibroblasts (CAFs), which control tumor behavior [16, 17].

Tumor budding at the invasive front is a reliable histopathological marker for estimating the aggressiveness of CRC and has important prognostic value [18-20]. The presence of "tumor budding" represents an independent predictor of tumor prognosis and metastasis in patients with both invasive and early CRC [14, 18, 19]. During cancer growth, tumors have been shown to actively create their own stroma, including desmoplastic stroma or CAF $[16,17]$. Although desmoplasia may defend against cancer invasion, the stroma may build up and support tumor invasion [16]. Although the role of tumor budding remains controversial in cancer progression, determination of the molecular events that occur during this phenomenon and in the surrounding area is essential to identify patients with aggressive disease [16, 17].

Many studies have reported the clinical significance of various molecular markers in CRC, including MMP-7 (matrilysin, pump-1), the laminin-5 $(\ln -5)$ y 2 chain and S-100A4 [7 - 13]. The expression of these biological markers has been suggested to be closely associated with invasion and progression [7-13]. Indeed, the expression of MMP-7, the $\ln -5$ y2 chain and S-100A4 is predictive of patient outcome [7-13].

Many researchers have attempted to identify the roles of clinicopathological variables, including venous and lymphatic invasion, tumor location, and tumor stage [1-6]. However, the associations between clinicopathological findings and these biomarkers at tumor budding sites have not been fully assessed. Therefore, we conducted a retrospective clinicopathological examination and immunohistological analysis of the expression of MMP7, the $\ln -5$ y 2 chain and S100A4 in CRC. The goal of this work was to evaluate the value of these factors in determining patient prognosis.

\section{Materials and Methods}

\section{Patients}

One hundred and six patients with CRC who underwent a colectomy between March 2006 and June 2008 were recruited from the Department of Diagnostic Pathology at Iwate Medical University. None of the patients received any preoperative therapy, such as chemo-radio therapy. The histological classification, tumor grade and stage were determined according to the typing scheme of the Japanese Classification of Colorectal Cancer [20]. The clinicopathological findings are summarized in Table 1.

Table 1. Demographic and pathological characteristics of patients with colorectal carcinoma

\begin{tabular}{cc}
\hline Variable & Frequency (\%) \\
\hline Male / Female & $65 / 41$ \\
Age (range) & $66(38-94)$ \\
Size (mm, range) & $57(15-120)$ \\
Locus & \\
Cecum & $6(5.7)$ \\
Ascending & $13(12.3)$ \\
Transverse & $4(3.8)$ \\
Descending & $6(5.7)$ \\
Sigmoid & $22(21.0)$ \\
Rectum & $55(51.9)$ \\
Differentiation & \\
Well & $23(21.7)$ \\
Moderately & $75(70.8)$ \\
Poor & $6(5.7)$ \\
Mucinous & $2(1.9)$ \\
Lymphatic invasion & \\
Low & $84(79.2)$ \\
High & $22(20.8)$ \\
Lymphatic invasion & \\
Low/negative & $80(75.5)$ \\
High & $26(25.5)$ \\
Stage classification & \\
III & $03(40.6)$ \\
\hline & $55(51.9)$ \\
\hline & $8(7.5)$ \\
\hline
\end{tabular}

well, well differentiated adenocarcinoma; moderate, moderately differentiated adenocarcinoma; poor, poorly differentiated adenocarcinoma; muc, mucinous adenocarcinoma. 
Microsatellite instability (MSI) is defined by the presence of one or more additional alleles in tumor samples compared to paired normal tissues, in accordance with international criteria. In the present study, MSI was excluded. Therefore, the CRC samples that we examined were microsatellite stable (MSS).

Formalin-fixed, paraffin-embedded tissue specimens from 106 patients with CRC (stages II-IV) who underwent curative surgery at Iwate Medical University were retrieved to assess the clinicopathological findings and immunohistochemistry. The study cohort consisted of CRC patients with typical adenocarcinoma histology, as confirmed by expert histopathologists. Our university Ethics Committee approved the study.

\section{Evaluation of lymphatic and vascular invasion in the CRC samples examined}

Lymphatic invasion was assessed by examining the histological features of normal lymphatic vessels. Positive lymphatic invasion was only identified when the cancer cells were floating within an endothelial-lined lymphatic channel that stained positively with anti-podoplanin antibody (D2-40, Dako). Vascular invasion was determined to be positive when the cancer cells were embedded within a vascular wall that was surrounded by elastic fiber (as confirmed by Verhoeff-van Gieson stain). Lymphatic invasion and vascular invasion were classified according to the Japanese Classification of Colorectal Cancer $(-, 1+, 2+$ and $3+)$ [20]. In the present study, whereas - and $1+$ invasion were regarded as the negative group, $2+$ and $3+$ invasion were assigned to the positive group.

\section{Study design}

This was a retrospective cohort study of patients diagnosed with CRC who underwent primary surgical resection at Iwate Medical University. No matching or stratification was used. The end of the follow-up period was January 18, 2013, death, or the date of loss to follow-up. The following criteria were used to exclude patients from the study cohort: previously treated with any anticancer therapy; impaired heart, lung, liver, or kidney function; previous malignant disease; and death from diseases other than CRC or from unexpected events. The median duration of follow-up for living patients was 5.5 years (range, 5-7 years). The objective of this retrospective analysis was to assess the associations between various prognostic factors and patient survival.

\section{Post-operative chemotherapy}

Post-operative chemotherapy was administered to 59 of 106 patients with CRC (55.7\%). The remaining patients did not undergo post-operative chemotherapy (47 of 106 patients, 44.3\%). Chemotherapeutic regimens included UFT, 5FU/LV and 5FU. The detailed results are shown in the Supplementary Table 1.

\section{Tissue microarrays}

On the hematoxylin and eosin (H\&E)-stained tumor tissue slides, a representative area of tumor budding at the invasive front was selected, and a corresponding spot was marked on the surface of a paraffin block. In the present study, the term "tumor invasive front" was defined as the deepest region in a given histological section. Using a biopsy needle, we extracted the selected area, and a 3-mm tissue core was placed into a $6 \times 5$ array. Each tissue core was assigned a unique tissue microarray location number, which was linked to a database that contained the other clinicopathological data.

\section{Immunohistochemistry}

We examined the expression of well-known prognostic markers in CRC using a tissue microarray (TMA). Antigen retrieval and staining with monoclonal antibodies against MMP7 (clone 141-7B2, Daiichi Fine Chemical, Japan), ln-5 y2 chain (clone 4G1, Dako, Denmark) and S100A4 (polyclonal, Dako, Denmark) was performed with an automatic immunostainer (Dako, Envision System, Denmark). In the present study, double immunostaining of pan-cytokeratin (CK, AE1/AE3, Dako, Denmark) and the markers (MMP7, the $\ln -5$ y2 chain and S100A4) was performed to identify epithelial cells in the tumor budding areas and surrounding fibroblasts (stromal cells). Briefly, for antigen retrieval, an ethylenediaminetetraacetic acid (EDTA) buffer ( $\mathrm{pH}$ 9.0) was used. To obtain a brown stain for the first antibody, we performed immunostaining using a Dako Envision+ system, followed by heat treatment for $10 \mathrm{~min}$ at $100^{\circ} \mathrm{C}$ and a $30-\mathrm{min}$ reaction time with anti-MMP7, anti-ln-5 Y2 chain and anti-S100A4 antibodies at room temperature. Thereafter, a denaturing solution (Biocare Medical) was added to terminate the above-mentioned $\mathrm{Ag}$ - $\mathrm{Ab}$ reaction before introducing the second mouse antibody (pancytokeratin; AE1/AE3, Dako, Denmark), and this reaction was allowed to proceed for $5 \mathrm{~min}$ at room temperature. Anti-cytokeratin (AE1/AE3, Dako) was then added and allowed to react for $30 \mathrm{~min}$ at room temperature, followed by reaction with the secondary antibody, MACH 2 Double Stain 1, and an alkaline phosphatase coloring agent, PermaRed/AP (Diagnostic Bio Systems, CA), which is a red-colored stain. Hematoxylin was used as the counterstain.

The percentage of positively stained cells was 
estimated with an image analysis system (Cell and Tissue Analysis, Leica, Germany) and graded as follows: grade 0 , no staining; grade $1,1-10 \%$ positive cells; grade 2, $11-50 \%$ positive cells; and grade 3, $51-100 \%$ positive cells. In the present study, samples exhibiting staining exceeding grade 2 were defined as positive.

Two pathologists (ST and UN) performed independent evaluations of the staining. If there was disagreement between the two pathologists, they reviewed the slides together, and consensus was obtained.

\section{Statistical analysis}

For statistical analysis of the expression of MMP7, the $\ln -5$ 2 chain and S100A4 in CRC and their associations with various clinicopathological factors and overall survival, we used the $\chi^{2}$-test, Fisher's exact test and the Mann-Whitney $U$-test with a $2 \times 2$ table to compare the categorical data. Overall patient survival was calculated from the date of the surgery to the date of the last follow-up or patient death. A univariate survival analysis was performed according to the Kaplan-Meier method, and survival was compared with the log-rank test. A multivariate analysis was conducted with the Cox proportional hazards regression model. The level of significance was $P<0.05$, and the confidence interval $(\mathrm{CI})$ was determined at the 95\% level. The statistical analyses were performed with the JMP 10.0 software package (SAS Institute, Inc., Cary, NC, USA) for Windows.

\section{Results}

\section{Clinicopathological findings in CRC}

Ninety-eight cases (92.5\%) showed a differentiated phenotype, whereas 8 cases $(7.5 \%)$ showed a less-differentiated phenotype. The welland moderately differentiated types were classified as differentiated. The less-differentiated types included mucinous adenocarcinoma and solid-type adenocarcinoma (excluding medullary carcinoma).

\section{Expression of MMP7 at the tumor budding site}

MMP7 was consistently expressed in the cytoplasm of the tumor cells and in the tumor budding areas, but was not detected in most of the stromal cells. The expression of MMP7 was found in 70 of the 106 CRC samples $(66.0 \%)$.

\section{Expression of the In-5 y2 chain at the tumor budding site}

Expression of the $\ln -5$ y 2 chain was commonly observed in the cytoplasm of the tumor cells. In addition, the $\ln -5 \gamma 2$ chain was expressed in the tumor budding nests but not in the stromal cells. The expression of the $\ln -5$ y 2 chain was noted at a low frequency at the tumor budding sites (9 of the 106 CRC samples, $8.5 \%$ ).

\section{Expression of S100A4 in the tumor budding nest and surrounding stromal cells}

Although S100A4 showed cytoplasmic and nuclear staining in the cancer cells, the nuclear S100A4 staining was considered positive because cytoplasmic expression was often observed in the non-neoplastic component. In the present study, expression in the epithelial and stromal cells was assessed separately. The expression of S100A4 in the stromal fibroblasts did not differ from that in the epithelial cancer cells. Both stromal and epithelial expression within the same histological section was detected frequently in the tumor budding area. Based on the criteria defined in this study, 44 of the 106 tumors $(41.5 \%)$ were classified as stromal fibroblast-positive, whereas 23 of the epithelial compartments of the 106 tumors $(21.7 \%)$ were classified as cancer cell-positive. Representative images showing the expression of MMP7, the $\ln -5 \gamma^{2}$ chain and S100A4 in the tumor budding areas are shown in Figure 1.

\section{Survival analyses with the clinicopathological findings and the expression of MMP7, the In-5 Y2 chain and S100A4}

Kaplan-Meier analyses were performed to determine and compare the overall 5-year survival rates. Tumor location, a less-differentiated histological type, lymphovascular invasion and tumor stage were correlated with overall survival (Figures 2 and 3). In addition, overall survival was associated statistically with the expression of S100A4 and the $\ln -5$ y 2 chain, with the latter divided into expression by epithelial or stromal cells (Figure 4). To determine whether the clinicopathological findings and biomarkers listed in Table 2 are independent predictors of survival among patients with CRC, we used a univariate analysis for preliminary screening of the variables, followed by a stepwise logistic regression of the risk of mortality with the significant univariate predictors. The univariate analysis (Table 2a) identified 8 factors associated with increased mortality in patients with CRC, including the tumor location, histological type, degree of lymphatic invasion, vascular invasion, tumor stage, neoplastic epithelial expression of S100A4, stromal cell expression of S100A4 and expression of the $\ln -5 \gamma 2$ chain. The presence/absence of chemotherapy was not correlated with patient survival in CRC. 

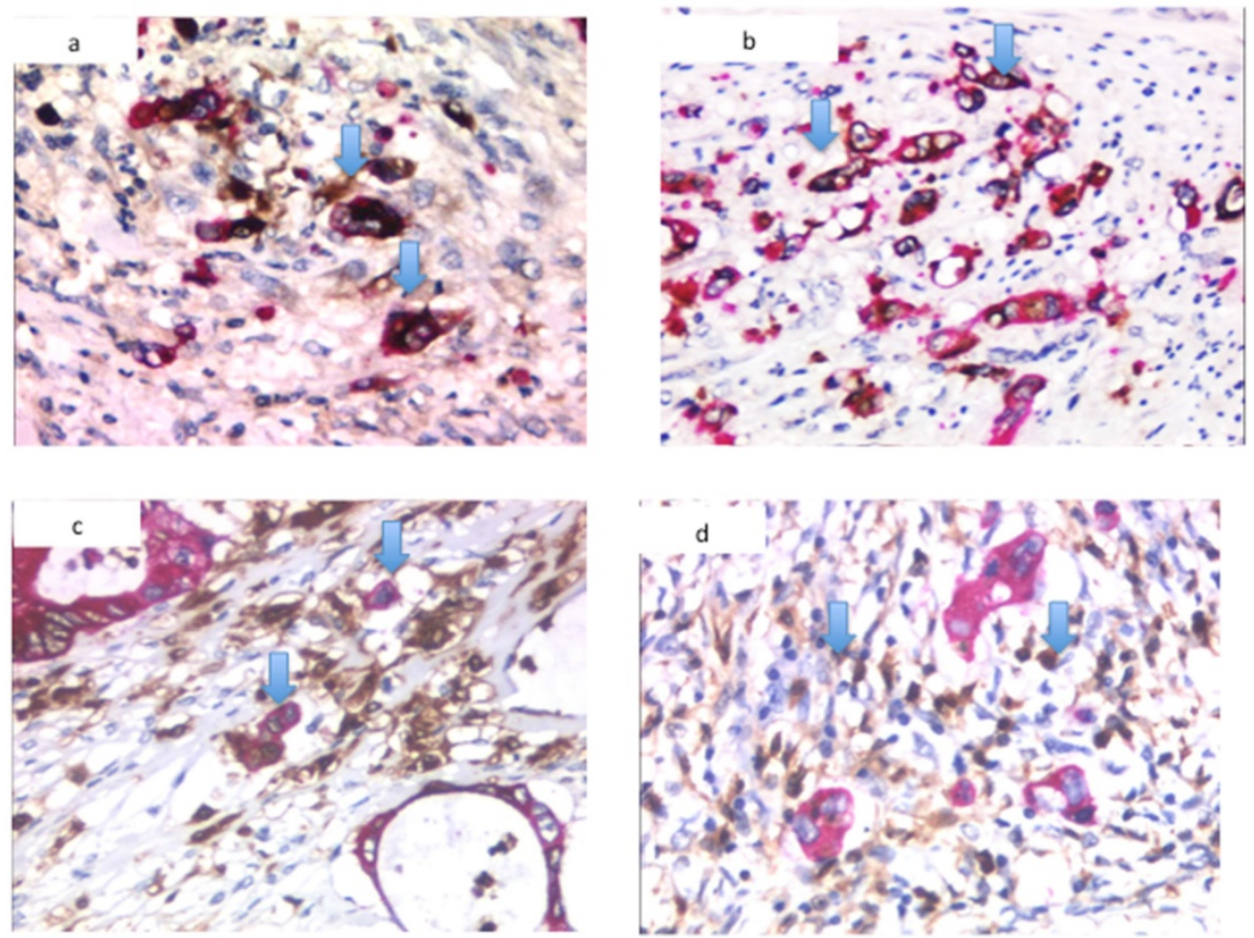

Figure 1. Immunohistochemical detection of CRC markers. (a) Immunocytochemical features of MMP7 expression, (b) In-5 Y2 chain expression and (c) S100A4 expression at a tumor budding site. (d) Stromal expression of S100A4 at a tumor budding site. Arrowheads indicate protein expression.

a

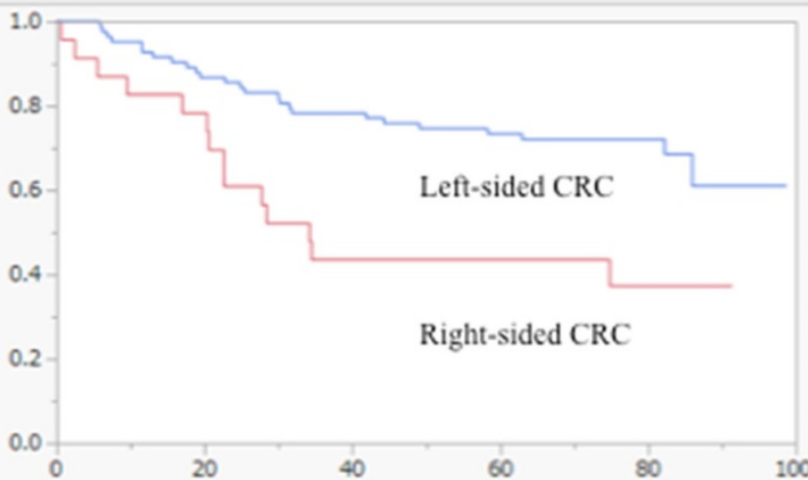

b

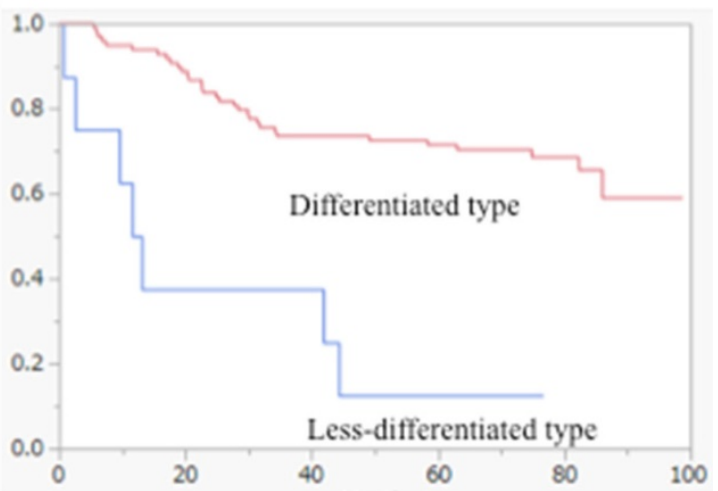

c

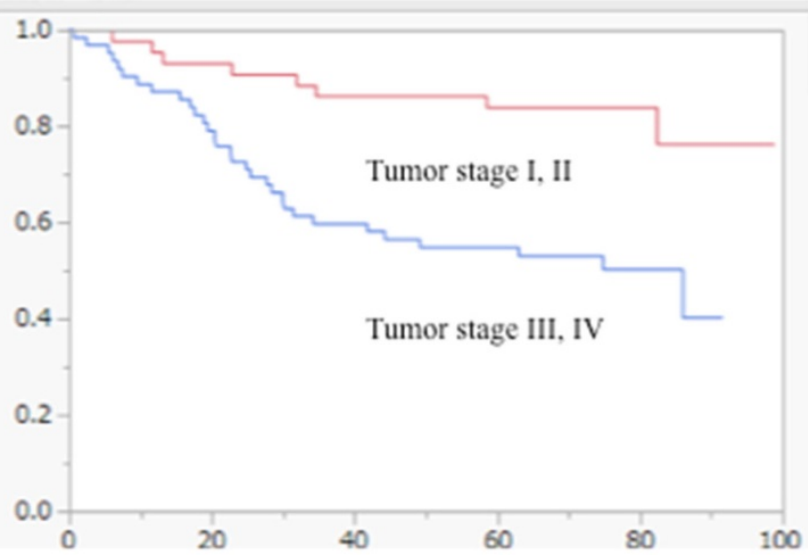

Figure 2. Kaplan-Meier survival analyses of tumor location, histology and stage in the cohort of patients with CRC (log-rank test). (a) Tumor location was statistically associated with overall patient survival $(p=0.0021)$. (b) Tumor histology was correlated with overall patient survival $(p<0.0001)$. (c) The overall survival rates of patients with tumor stages III and IV were significantly shorter than those of patients with stages I and II $(p=0.0009)$. 


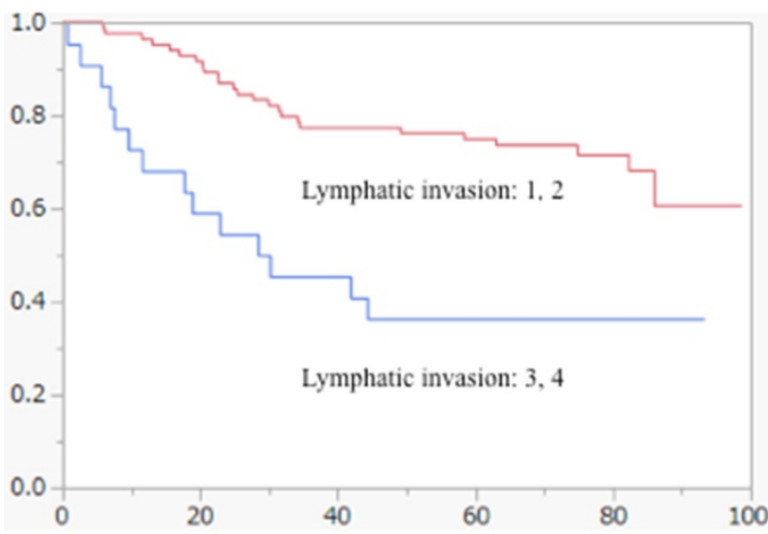

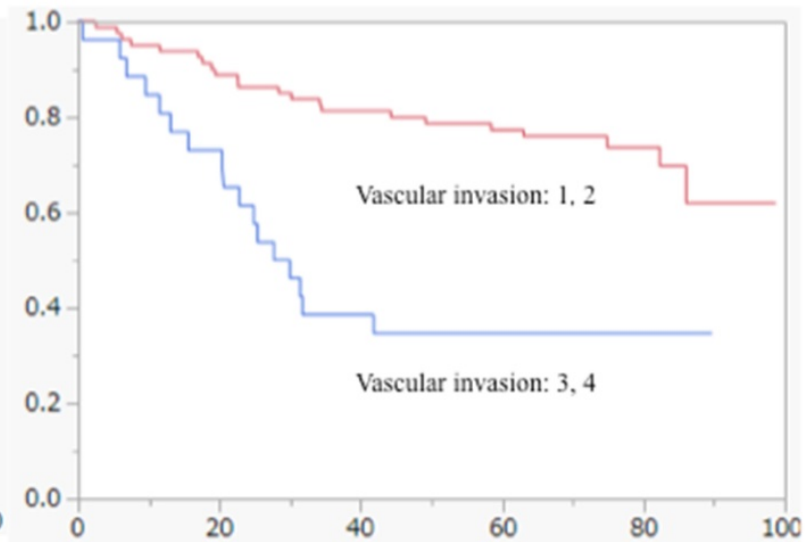

Figure 3. Kaplan-Meier survival analyses of lymphovascular invasion in the cohort of patients with CRC (log-rank test). (a) Lymphatic invasion was statistically associated with overall patient survival $(p=0.0002)$. (b) Vascular invasion was correlated with overall patient survival $(p=0.001)$.
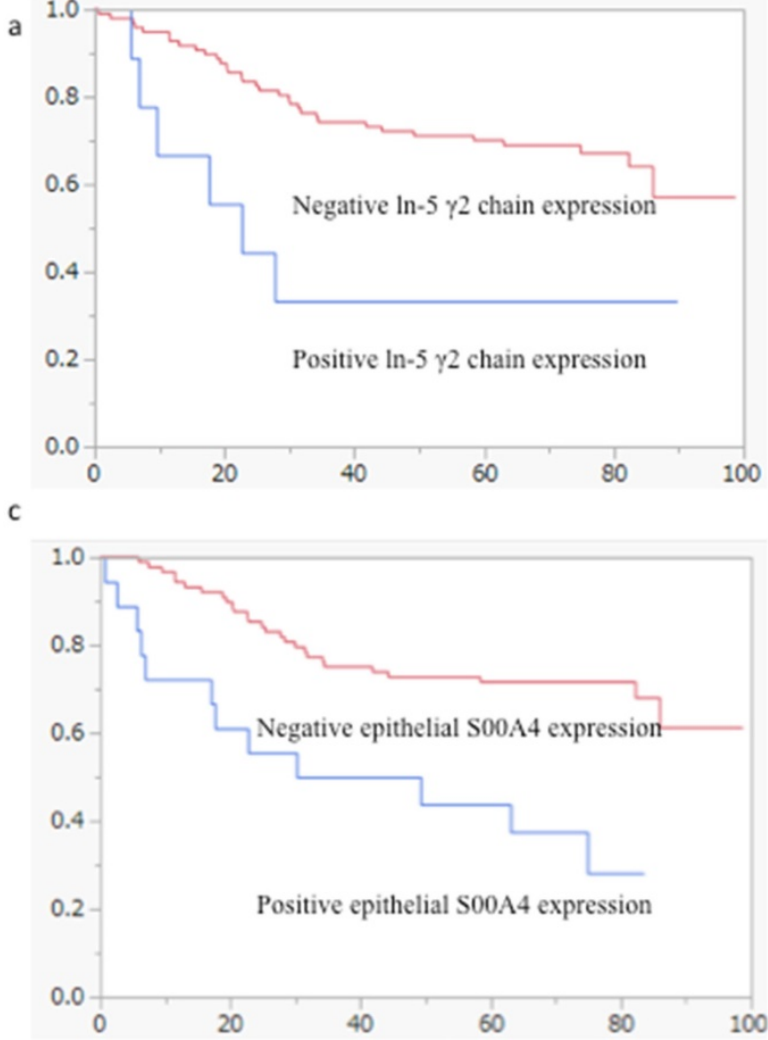

b

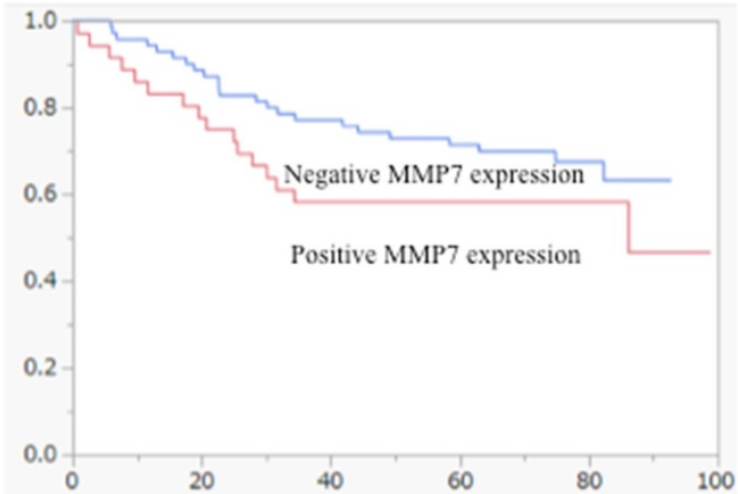

d

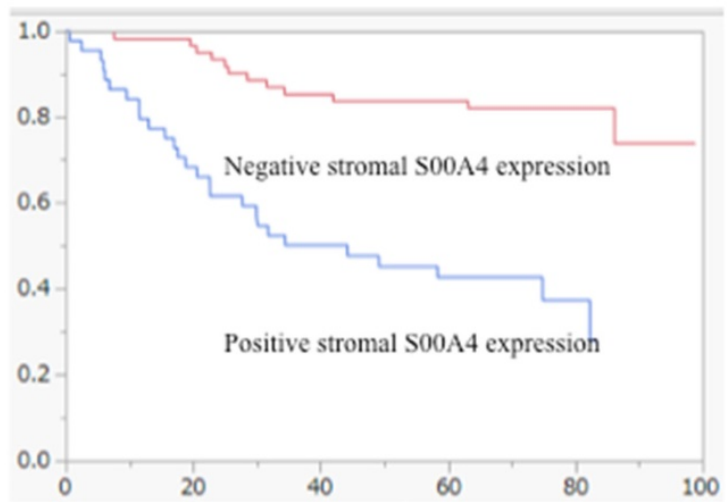

Figure 4. Kaplan-Meier survival analyses of the expression of the In-5 y2 chain, MMP7 and S100A4 in the cohort of patients with CRC (log-rank test). (a) The overall survival of patients with positive $\ln -5 \mathrm{y} 2$ chain expression was significantly shorter than that of patients with negative expression ( $P=0.0055)$. (b) Overall patient survival was not associated with MMP7 expression. (c) The overall survival of patients with positive S100A4 epithelial expression was significantly shorter than that of patients with negative expression $(p=0.0073)$. ( $(d)$ The overall survival of patients with positive S100A4 stromal expression was significantly shorter than that of patients with negative expression $(p=0.0008)$.

Table $2 \mathrm{~b}$ lists the 5 factors that were retained in the multivariate logistic regression analysis. We found that tumor location (HR, 0.38; 95\% CI, 0.18-0.83), lymphatic and vascular invasion $(\mathrm{HR}, 3.23 ; 95 \% \mathrm{CI}$, 1.28-7.76 and HR, 4.30; 95\% CI, 2.04-8.97, respectively), tumor stage (HR, 3.40; 95\% CI, 1.57-8.25) and stromal cell expression of S100A4 (HR, $6.37 ; 95 \%$ CI 2.65-16) remained significant predictors of mortality, even after controlling for the other variables. Histological type (HR, 1.68; 95\% CI, 0.54-4.92), epithelial expression of S100A4 (HR, 1.98; $95 \% \mathrm{CI}, 0.84-4.58)$ and expression of the $\ln -5 \gamma 2$ chain (HR, 0.66; 95\% CI, 0.21-1.78) were not factors contributing to mortality after adjusting for the effects of the other factors. These results are summarized in Table 2. 
Table 2. Cox univatiate and multivariate analysis for overall survival in colorectal cancer

\begin{tabular}{|c|c|c|c|c|c|c|c|}
\hline \multirow{2}{*}{\multicolumn{2}{|c|}{ Variable (number) }} & \multicolumn{3}{|c|}{ Univariate analysis } & \multicolumn{3}{|c|}{ Multivariate analysis } \\
\hline & & Hazard ratio & $95 \%$ confidence interval & P value & Hazard ratio & $95 \%$ confidence interval & $P$ value \\
\hline Sex & $\begin{array}{l}\text { Male (65) } \\
\text { Female (41) }\end{array}$ & 0.98 & $0.50-1.85$ & 0.9503 & & & \\
\hline Age & $\begin{array}{l}60 y>(25) \\
60 y \leqq(81)\end{array}$ & 1.62 & $0.76-4.01$ & 0.2417 & & & \\
\hline Size $(\mathrm{mm})$ & $\begin{array}{l}50>(41) \\
50 \leqq(65)\end{array}$ & 1.08 & $0.57-2.11$ & 0.8148 & & & \\
\hline Locus & $\begin{array}{l}\text { Right (23) } \\
\text { Left (83) }\end{array}$ & 0.37 & $0.19-0.73$ & $0.0021^{*}$ & 0.38 & $018-0.83$ & 0.0129 \\
\hline Histology & $\begin{array}{l}\text { Differentiated ( } 98) \\
\text { Less differentiated ( } 8 \text { ) }\end{array}$ & 5.74 & $2.30-12.45$ & $<0.0001^{*}$ & 1.68 & $0.54-4.92$ & 0.0347 \\
\hline $\begin{array}{l}\text { Lymphatic } \\
\text { invasion }\end{array}$ & $\begin{array}{l}0 \cdot 1(84) \\
2 \cdot 3(22)\end{array}$ & 3.26 & $1.64-6.21$ & $0.0002^{*}$ & 3.23 & $1.28-7.76$ & 0.0135 \\
\hline $\begin{array}{l}\text { Vascular } \\
\text { invasion }\end{array}$ & $\begin{array}{l}0 \cdot 1(80) \\
2 \cdot 3(26)\end{array}$ & 3.44 & $1.79-6.50$ & $0.001^{*}$ & 4.30 & $2.04-8.97$ & 0.0004 \\
\hline Stage & $\begin{array}{l}\text { I· II (44) } \\
\text { III } \cdot \text { IV (62) }\end{array}$ & 3.43 & $1.66-8.04$ & $0.0009^{*}$ & 3.40 & $1.57-8.25$ & 0.0014 \\
\hline $\begin{array}{l}\text { Grade of tumor } \\
\text { budding }\end{array}$ & $\begin{array}{l}\text { low }(81) \\
\text { high }(25)\end{array}$ & 1.17 & $0.56-2.30$ & 0.6540 & & & \\
\hline S100A4 (tumor cell) & $\begin{array}{l}\text { negative (83) } \\
\text { positive (23) }\end{array}$ & 2.388 & $1.21-4.54$ & $0.0073^{*}$ & 1.98 & $0.84-4.58$ & 0.1145 \\
\hline S100A4 interstitial & $\begin{array}{l}\text { negative (62) } \\
\text { positive (44) }\end{array}$ & 4.56 & $2.32-9.62$ & $<0.001^{*}$ & 6.37 & $2.65-16$ & 0.0001 \\
\hline MMP7 & $\begin{array}{l}\text { negative (36) } \\
\text { positive (70) }\end{array}$ & 0.65 & $0.34-1.25$ & 0.1775 & & & \\
\hline $\begin{array}{l}\text { Laminin- } \\
5 \gamma 2 \text { Chain }\end{array}$ & $\begin{array}{l}\text { negative( }(97) \\
\text { positive (9) }\end{array}$ & 3.23 & $1.21-7.26$ & 0.0055 & 0.66 & $0.21-1.79$ & 0.4393 \\
\hline Chemotherapy & $\begin{array}{l}\text { done (59) } \\
\text { not done (47) }\end{array}$ & 1.33 & $0.71-2.52$ & 0.3650 & & & \\
\hline
\end{tabular}

\section{Discussion}

Previous studies have shown that tumor budding at the cancer invasive front plays an essential role in tumor invasion $[14,18,19]$. The role of tumor budding is based on the molecular alterations of the underlying tumor budding cells [21, 22]. Assessment of the extent of tumor budding at the invasive front has been reported to predict patient prognosis or tumor metastasis in CRC $[14,18]$. The latter requires the detection of tumor budding foci by histological examination of conventionally H\&E-stained sections $[14,18]$. However, detecting tumor buds accurately is difficult, because they may be very small and have confusing histological features that resemble those of inflammatory or interstitial cells [19]. In the present study, immunohistochemical detection was performed to objectively assess tumor budding at the tumor's invasive front. The goal of this analysis was to examine the molecular alterations of the underlying budding process and the surrounding stroma (CAFs).

In the present study, tumor location and less-differentiated histological cell type affected patient prognosis in CRC. Left-sided CRC is characterized by frequent p53 mutations and chromosomal instabilities associated with the malignant phenotype of cancer cells [23, 24]. In addition, less-differentiated histological cell types (poorly differentiated and mucinous carcinoma) are related to patient prognosis [25]. The prognosis of patients with poorly differentiated colorectal adenocarcinomas is typically reported to be poor and less favorable than that of patients with well- or moderately differentiated adenocarcinomas [25]. Although previous studies have shown that the frequency of poorly differentiated colorectal adenocarcinomas is very low [25], this histological type is important in the prediction of patient prognosis in CRC. The association between a poorly differentiated type and poor overall survival may indicate that the molecular alterations are attributable to a high frequency of chromosomal aberrations [25].

The TNM stage is defined by the tumor invasive depth, lymph node metastasis and distant metastasis, and it correlates with patient prognosis. Although lymphatic and vascular invasion are not included in 
this scheme [25], these factors are believed to be closely associated with patient survival [3-5]. In the present study, lymphatic and vascular invasion remained significant prognostic indicators in the multivariate analysis. In particular, vascular invasion in CRC correlated well with patient survival, representing an independent prognostic factor after curative resection [4-6]. The present finding suggests that vascular invasion can be used to evaluate tumor aggressiveness and estimate patient survival, irrespective of the actual number of positive lymph nodes. However, considerable inter- or intra-observer variations exist among pathologists' judgments of lympho-vascular invasion [26]. Although previous studies have shown that agreement was not improved by the use of immunohistochemical or elastin stains, we believe that our evaluation of lympho-vascular invasion affects the clinical assessment of patient prognosis.

MMPs can degrade most components of the extracellular matrix (ECM) [27]. Although MMPs share substrates, they have different specificities. MMP7 is one of the most important molecules in the MMP family, because it has the ability to degrade the vascular basement membrane, indicating its potential to facilitate hematogenous metastasis [27]. Previous studies have suggested that the activation of MMP7 is a critical molecular step leading to liver metastases and lymph node metastasis in CRC [27, 28]. Our results showed that the overexpression of MMP7 occurs frequently in the tumor budding nest. This finding substantiates a previous report that MMP7 expression in cancer cells was quite often observed at the invasive front, suggesting that it may be induced by tumor-host interactions [27]. Although this finding is considered to be very important in tumor progression, our results suggest that overexpression of MMP7 is not associated with patient prognosis. The reason underlying these contrasting results remains unknown, but it may depend on the expression of MMP7 at the tumor budding site. Despite this limitation, MMP7 expression by tumor cells may provide useful information regarding patient prognosis or tumor invasion.

The laminins constitute a group of matrix proteins localized at the basement membrane, where they are involved in cell adhesion [9, 13, 28]. The laminin family consists of heterodimers composed of $\alpha, \beta$, and $\gamma$ subunits (28). A previous study revealed that the expression of the $\ln -5 \gamma 2$ chain increases during carcinogenesis $[9,13,28]$ and is also associated with tumor progression $[9,13,28]$. This finding is in agreement with our results, which show that $\ln -5 \gamma^{2}$ chain expression was upregulated during the progression of human CRC. However, based on the present study, we cannot prove the prognostic value of $\ln -5$ y2 chain expression at the invasive front of CRC. Our results also revealed that expression of the $\ln -5$ y2 chain occurred at a low frequency in tumor budding. We suggest that detection of the $\ln -5 \gamma^{2}$ chain as a tumor prognostic marker in CRC has limited value.

In recent years, a number of studies have examined the correlation of S100A4 expression with the survival and prognosis of CRC patients [11, 29-32]. The overexpression of S100A4 plays a role in cancer progression by enhancing tumor invasion and, thereby, the metastatic potential of CRC [29-32]. Our results revealed that, although epithelial and stromal expression of S100A4 correlated with patient prognosis in the univariate analysis, only stromal expression of S100A4 was closely associated with patient outcome in the multivariate analysis. This finding reminds us that overall S100A4 expression that does not distinguish epithelial expression from stromal expression helps to predict the prognosis of patients with CRC. However, this finding represents an important concept, in that CAFs play an important role in the progression of CRC. Our results support the theory that CAFs, which are commonly observed in tissues surrounding tumor budding, are determining factors in tumor invasion.

The underlying molecular process that associates S100A4 expression with patient prognosis remains unclear. Considerable evidence indicates that S100A4 expression by cancer cells alters their adhesive properties, possibly by remodeling the ECM and promoting the redeployment of adhesion-mediating molecules [30, 32]. Additionally, the induction of S100A4 may be linked to the downregulation of E-cadherin and cytoskeletal dysregulation [31]. This finding is supported by data showing that the expression of E-cadherin, an epithelial adhesion molecule, is inversely correlated with the expression of S100A4 [31]. In addition, p53 function may be inhibited in cells expressing S100A4 [29]. If so, the genetic instability of cancer cells or CAFs producing S100A4 may be enhanced by p53 dysfunction.

Although the role of CAFs has received attention in terms of colorectal carcinogenesis, recent studies have shown that interstitial components that are associated with cancer tissue, such as vessels and lymphocytes, play an important role in the progression of CRC. Jiang et al. showed that lymphocytes and tumor cells are connected through membrane fusion to form tumor-lymphocyte chimeras (TLCs) that allow the tumor stem cell to take advantage of the lymphocyte's ability to migrate and destroy cell barriers to invade the vessel channel and to travel to distant organs (33). In addition, Jiang et al. 
also revealed that tube-like structures with co-expression of D2-40 and CD34, both of which are associated with CK19-positive tumor cells that are thought to be stem cell markers, are related to the development of newly formed cancer-associated vasculature. This finding suggested that tumor stem cells within the epithelial and stromal components contribute equally and coordinately to the morphogenesis of new vasculature, which constitutes the basis for the unique morphologic and immunohistochemical features of newly formed vasculature (34). Although these findings suggest the unique hypothesis that tumor cells use vessels and lymphocytes that appear normal histologically to promote the development of tumor cells, our findings indicated that stromal fibroblasts expressing S100A4 contribute to poor outcomes in patients with CRC.

Our characterization of these CRC prognostic markers (including S100A4) is somewhat promising. However, for the following reasons, these findings must be further confirmed by large, prospective studies. First, the present study was retrospective. A prospective analysis would be required to prove the utility of a given marker. Second, it is possible that other, unknown confounders may have biased the data [30,31]. Third, establishing the cut-off values for S100A4 expression was a significant challenge. The discrepancies among the studies performed to date can be attributed to the use of different cut-offs [29, 30, 31]. Indeed, providing an exact definition of "high" vs. "low" or "positive" vs. "negative" expression was difficult because of the different detection methods used. Therefore, future studies addressing this issue should use a consistent definition for the expression and the same detection criteria. Fourth, whether an S100A4-positive cell is a cancer cell or a stromal cell is an important factor in determining the prognosis of a patient with CRC. Although different interpretations of the role of S100A4 expression in cancer invasion exist, our results provide novel findings for the prediction of patient survival. Finally, it is well recognized that post-operative chemotherapy is closely associated with patient survival. In the present study, 53.7\% of the patients with CRC underwent post-operative chemotherapy, but the presence/absence of chemotherapy was not correlated with patient survival. In addition, although 3 different chemotherapy regimens (UFT, 5FU/LV and 5FU) were used, the drugs they contain have common mechanisms of pharmacological action of inhibition of DNA synthesis. Therefore, we believe that it is unlikely that the difference in regimens had an influence on patient survival.

In conclusion, we examined tumor prognostic markers to elucidate their associations with adverse clinical outcomes. In CRC, the expression of S100A4 by stromal cells surrounding the tumor budding site represents a highly significant prognostic marker able to identify a subset of patients at high risk. This is a novel finding in that tumor budding, which is thought to be an important prognostic factor in CRC, is closely associated with the biological activation of cancer-associated fibroblasts that is seen surrounding tumor budding nests. In addition, vascular invasion is a strong indicator of patient prognosis in CRC. Although our results suggest that S100A4 could be a useful biological predictor of poor outcome, subsequent, large-scale, prospective studies are needed to confirm our results.

\section{Abbreviations}

CAF: cancer associated fibroblast; CRC: colorectal cancer; ECM: extracellular matrix; $\ln -5$ : laminin-5 (ln-5) ү2; MSI: microsatellite instability; MSS: microsatellite stable; TMA: Tissue Microarray; MMP7: matrix metalloproteinase-7; TNM: Tumor Node Metastasis.

\section{Supplementary Material}

Supplementary table 1.

http://www.jcancer.org/v08p1552s1.pdf

\section{Acknowledgments}

We gratefully acknowledge the technical assistance of members of the Department of Molecular Diagnostic Pathology at Iwate Medical University for their support.

\section{Author contributions}

T Sugai, who is the first and the corresponding authors, contributed to the preparation of the manuscript, including all aspects of data collection and analysis. $\mathrm{N}$ Yamada performed the experimental work and constructed the figures and tables. $M$. Eizuka performed the statistical analysis. K. Tsuchida, R. Sugimoto and M. Osakabe provided input during the preparation of the manuscript. N. Uesugi and $\mathrm{K}$. Ishida provided support for the experiments involving molecular technologies. K. Otsuka and A. Sasaki assisted with the clinical data and experiments. $\mathrm{T}$ Matsumoto discussed the important points of the manuscript.

\section{Competing Interests}

The authors have declared that no competing interest exists.

\section{References}

1. Mehrkhani F, Nasiri S, Donboli K, et al. Prognostic factors in survival of colorectal cancer patients after surgery. Colorectal Dis. 2009;11:157-61. 
2. von Winterfeld M, Hoffmeister M, Ingold-Heppner B, et al. Frequency of therapy-relevant staging shifts in colorectal cancer through the introduction of pN1c in the 7th TNM edition. Eur J Cancer. 2014;50:2958-65.

3. Jiang PC, Zhu L, Fan Y, et al. Clinicopathological and biological significance of cripto overexpression in human colon cancer. World J Gastroenterol. 2013;19 (46):8630-7.

4. Akishima-Fukasawa Y, Ishikawa Y, Akasaka Y, et al. Histopathological predictors of regional lymph node metastasis at the invasive front in early colorectal cancer. Histopathology. 2011;59:470-81.

5. Courtney ED, West NJ, Kaur C, et al. Extramural vascular invasion is an adverse prognostic indicator of survival in patients with colorectal cancer. Colorectal Dis. 2009;11:150-6.

6. McClelland D, Murray GI. A Comprehensive Study of Extramural Venous Invasion in Colorectal Cancer. PLoS One. 2015;10:e0144987.

7. Polistena A, Cucina A, Dinicola S, et al. MMP7 expression in colorectal tumours of different stages. In Vivo. 2014;28:105-10.

8. Karamitopoulou E, Zlobec I, Panayiotides I, et al. Systematic analysis of proteins from different signaling pathways in the tumor center and the invasive front of colorectal cancer. Hum Pathol. 2011;42:1888-96.

9. Masaki $T$, Matsuoka $H$, Sugiyama $M$, et al. Laminin-5 gamma2 chain expression as a possible determinant of tumor aggressiveness in T1 colorectal carcinomas. Dig Dis Sci. 2003;48:272-8.

10. Weidle UH, Birzele F, Krüger A. Molecular targets and pathways involved in liver metastasis of colorectal cancer. Clin Exp Metastasis. 2015;32:623-35.

11. Niu Y, Wang L, Cheng C, et al. Increased expressions of SATB1 and S100A4 are associated with poor prognosis in human colorectal carcinoma. APMIS. 2015;123:93-101.

12. Sun DW, Zhang YY, Qi Y, et al. Prognostic significance of MMP-7 expression in colorectal cancer: a meta-analysis. Cancer Epidemiol. 2015;39:135-42.

13. Aoki S, Nakanishi Y, Akimoto S, et al. Prognostic significance of laminin-5 gamma2 chain expression in colorectal carcinoma: immunohistochemical analysis of 103 cases. Dis Colon Rectum. 2002;45:1520-7.

14. Puppa G, Senore C, Sheahan K, et al. Diagnostic reproducibility of tumour budding in colorectal cancer: a multicentre, multinational study using virtual microscopy. Histopathology. 2012;61:562-75.

15. Ueno H, Shinto E, Kajiwara Y, et al. Prognostic impact of histological categorisation of epithelial-mesenchymal transition in colorectal cancer. $\mathrm{Br} \mathrm{J}$ Cancer. 2014;111:2082-90.

16. Galván JA, Helbling M, Koelzer VH, et al. TWIST1 and TWIST2 promoter methylation and protein expression in tumor stroma influence the epithelial-mesenchymal transition-like tumor budding phenotype in colorectal cancer. Oncotarget. 2015;6:874-85.

17. Tommelein J, Verset L, Boterberg $\mathrm{T}$, et al. Cancer-associated fibroblasts connect metastasis-promoting communication in colorectal cancer. Front Oncol. 2015;5:63.

18. Ueno H, Murphy J, Jass JR, et al. Tumour 'budding' as an index to estimate the potential of aggressiveness in rectal cancer. Histopathology. 2002;40:127-32.

19. Shinto $\mathrm{E}$, Mochizuki $\mathrm{H}$, Ueno $\mathrm{H}$, et al A novel classification of tumour budding in colorectal cancer based on the presence of cytoplasmic pseudo-fragments around budding foci. Histopathology. 2005;47:25-31.

20. Japanese Society for Cancer of the Colon and Rectum. Japanese Classification of Colorectal Carcinoma, Second English Edition. Kanehara Co.: Tokyo. 2009: 30-63.

21. Zlobec I, Lugli A. Epithelial mesenchymal transition and tumor budding in aggressive colorectal cancer: tumor budding as oncotarget. Oncotarget. 2010;1:651-61.

22. Lamouille $\mathrm{S}, \mathrm{Xu}$ J, Derynck R. Molecular mechanisms of epithelial-mesenchymal transition. Nat Rev Mol Cell Biol. 2014;15:178-96.

23. Sugai T, Habano W, Jiao Y-F, et al. Analysis of molecular alterations in leftand right-sided colorectal carcinomas reveals distinct pathways of carcinogenesis: proposal for new molecular profile of colorectal carcinomas. J Mol Diagn 2006; 8: 193-201.

24. Cancer Genome Atlas Network. Comprehensive molecular characterization of human colon and rectal cancer. Nature 2012; 487: 330-337.

25. Snover D, Ahnen DJ, Burt R et al. Serrated polyps of the colon and rectum and serrated ("hyperplastic") polyposis. In: Bosman FT, Carneiro F, Hruban RH, et al, (eds) WHO Classification of Tumours Pathology and genetics Tumours of the digestive system, 4th edn. Berlin: Springer-Verlag, 2010.

26. Kojima M, Shimazaki $H$, Iwaya $K$, et al. Pathological diagnostic criterion of blood and lymphatic vessel invasion in colorectal cancer: a framework for developing an objective pathological diagnostic system using the Delphi method, from the Pathology Working Group of the Japanese Society for Cancer of the Colon and Rectum. J Clin Pathol. 2013;66:551-8.

27. Fanjul-Fernández $M$, Folgueras AR, Cabrera $S$, et al. Matrix metalloproteinases: evolution, gene regulation and functional analysis in mouse models. Biochim Biophys Acta. 2010;1803:3-19.

28. Lenander C, Roblick UJ, Habermann JK, et al. Laminin 5 gamma 2 chain expression: a marker of early invasiveness in colorectal adenomas. Mol Pathol. 2003;56:342-6.

29. Kwak JM, Lee HJ, Kim SH, et al. Expression of protein S100A4 is a predictor of recurrence in colorectal cancer. World J Gastroenterol. 2010;16:3897-904.

30. Sack U, Walther W, Scudiero D, et al. S100A4-induced cell motility and metastasis is restricted by the Wnt/ $\beta$-catenin pathway inhibitor calcimycin in colon cancer cells. Mol Biol Cell. 2011;22:3344-54.
31. Lee SJ, Choi SY, Kim WJ, et al. Combined aberrant expression of E-cadherin and S100A4, but not $\beta$-catenin is associated with disease-free survival and overall survival in colorectal cancer patients. Diagn Pathol. 2013;8:99.

32. Boye K, Nesland JM, Sandstad B, et al. EMMPRIN is associated with S100A4 and predicts patient outcome in colorectal cancer. Br J Cancer. 2012;107:667-74.

33. Jiang B, Mason J, Jewett A, et al Cell budding from normal appearing epithelia: a predictor of colorectal cancer metastasis? In J Biol Sci. 2013;9:119-33.

34. Jiang B, Mason J, Jewett A, et al. Tube-like structures with co-expression of D2-40 and CD34: newly formed vasculatures? Int J Biol Sci. 2012;8:1206-16. 